Dear Author,

Please, note that changes made to the HTML content will be added to the article before publication, but are not reflected in this PDF.

Note also that this file should not be used for submitting corrections. 


\title{
Endocytosis of fluorescent cyclodextrins by intestinal Caco-2 cells and its role in paclitaxel drug delivery
}

\author{
3 Q1 Katalin Réti-Nagy ${ }^{\mathrm{a}}$, Milo Malanga ${ }^{\mathrm{b}}$, Éva Fenyvesi ${ }^{\mathrm{b}}$, Lajos Szente $^{\mathrm{b}}$, György Vámosic $^{\mathrm{c}}$, \\ Judit Váradia , Ildikó Bácskaya ${ }^{a}$, Pálma Fehér ${ }^{a}$, Zoltán Ujhelyi ${ }^{a}$, Eszter Róka ${ }^{a}$, \\ Miklós Vecsernyés ${ }^{\mathrm{a}}$, György Balogh ${ }^{\mathrm{d}}$, Gábor Vasvári ${ }^{\mathrm{a}}$, Ferenc Fenyvesi ${ }^{\mathrm{a}, *}$ \\ a Department of Pharmaceutical Technology, University of Debrecen, H-4010 Nagyerdei St. 98, Debrecen, Hungary \\ ${ }^{\mathrm{b}}$ Cyclolab Cyclodextrin RED Laboratory Ltd., H-1097 Illatos St. 7., Budapest, Hungary \\ ${ }^{\mathrm{c}}$ Department of Biophysics and Cell Biology, University of Debrecen, H-4010 Egyetem Sq. 1., Debrecen, Hungary \\ d Gedeon Richter Plc., H-1103 Gyömröi út 19-21., Budapest, Hungary
}

A R T I C L E I N F O

\section{Article history:}

Received 17 July 2015

Received in revised form 14 October 2015

Accepted 16 October 2015

Available online $\mathrm{xxx}$

Chemical compounds studied in this article:

(2-Hydroxypropyl)- $\beta$-cyclodextrin

(PubChem CID: 44134771)

Random methyl- $\beta$-cyclodextrin (PubChem CID: 51051622)

Paclitaxel (PubChem CID: 36314)

Keywords:

Cyclodextrin

Endocytosis

Caco-2

Paclitaxel

\begin{abstract}
A B S T R A C T
Cyclodextrins are widely used excipients in pharmaceutical formulations. They are mainly utilized as solubilizers and absorption enhancers, but recent results revealed their effects on cell membranes and pharmacological barriers. In addition to the growing knowledge on their interaction with plasma membranes, it was confirmed that cyclodextrins are able to enter cells by endocytosis. The number of the tested cyclodextrins was limited, and the role of this mechanism in drug absorption and delivery is not known. Our aim was to examine the endocytosis of fluorescently labeled hydroxypropyl- $\beta$-cyclodextrin, random methyl- $\beta$-cyclodextrin and soluble $\beta$-cyclodextrin polymer, and the cellular uptake of the fluorescent paclitaxel derivative-random methyl- $\beta$-cyclodextrin complex. The studied cyclodextrin derivatives were able to enter Caco-2 intestinal cells and localized in vesicles in the cytoplasm, while their permeability was very limited through Caco-2 monolayers. We demonstrated for the first time that the fluorescent paclitaxel derivative and rhodamine-labeled random methyl- $\beta$-cyclodextrin were detected in the same intracellular vesicles after treating cells with their inclusion complex. These results indicate that the endocytosis of cyclodextrin complexes can contribute to drug absorption processes.
\end{abstract} (c) 2015 Published by Elsevier B.V.

\section{Introduction}

Cyclodextrins are widely used in drug formulations for solubilization of active pharmaceutical ingredients with rather lipophilic character and poor solubility. For the effective solubilization, drug molecules require proper cyclodextrin selected from a great variety of derivatives.

According to the ring size, cyclodextrins have three basic types: $\alpha-, \beta$ - and $\gamma$-cyclodextrins containing 6,7 or 8 glucopyranose units, respectively (Szejtli, 2004). Various derivatives of cyclodextrins can be formed by substitution of these rings. The number of published derivatives is more than 1500 . Production should be simple and cheap, the ring should keep its complexation capacity and the new product should have no toxic effects. In the industry

\footnotetext{
* Corresponding author at: Department of Pharmaceutical Technology, University of Debrecen, PO Box 78, H-4010 Debrecen, Hungary. Fax: +36 52411 600/54542. E-mail address: fenyvesi.ferenc@pharm.unideb.hu (F. Fenyvesi).
}

mainly methylated- (DIMEB, RAMEB), hydroxyalkylated- (HPBCD), sulfobutylated- (SBE-CD), acetylated- (acetyl- $\gamma C D)$ and branched (glucosyl-, maltosyl- $\beta C D$ ) cyclodextrins are produced. Safety studies revealed, that HPBCD and SBE-CD are well tolerated in humans and have no adverse effects on the kidneys or other organs (Stella and He, 2008). Cyclodextrin complexes with proper stability constants are able to improve the absorption and bioavailability of the complexed drug. There are numerous mechanisms published, which can explain this behavior.

The first mechanism is based on the solubility-increasing effect of cyclodextrins. The complexed lipophilic molecule can get hydrophilic properties by the cyclodextrin ring. In addition, the guest molecule can be transported through the unstirred water layer (UWL) directly to the biological membrane with the help of cyclodextrin. Viscous mucus membranes have an adsorbed, unstirred water layer on their surface which can be $100 \mu \mathrm{m}$ thick (Lennernas, 1998; Loftsson et al., 2007). Hydrophilic cyclodextrins can increase the drug transport only if the resistance of UWL on donor side is equal or higher than the resistance of membrane barrier 
(Loftsson et al., 2007; Masson et al., 1999). According to this mechanism, the complexed drug is carried to the membrane where the complex dissociates and only the free drug molecule penetrates through the membrane (Loftsson et al., 2005). In silico simulations confirmed that $\beta C D$ and $\beta C D$-involved assemblies confronting large 22 energy barriers to go across the membrane (Ren et al., 2015).

On the other hand if the binding force is too strong between the cyclodextrin and the guest molecule, only a small portion of the drug is released from the complex, resulting in lower bioavailability (Brewster et al., 2007).

The second action is based on the effect of cyclodextrins on intestinal epithelium. Lipophilic cyclodextrins (e.g., randomly methylated $\beta$-cyclodextrin) are able to decrease the barrier function, and by this the drug transport is increased through biological membranes (e.g., nasal mucus membrane). However, excess amount of hydrophilic cyclodextrin can decrease the penetration of drugs (Loftsson et al., 2007).

Beside the above mentioned effects, active transporter inhibition can be important as well. Many active transporter proteins (e.g., P-glycoprotein) can decrease the absorption of their substrates by pumping them back from the membrane to the lumen of the small intestine. Methylated- $\beta$-cyclodextrins are able to prevent the operation of the transporter proteins by reducing the cholesterol content of the membrane (Arima et al., 2004; Fenyvesi et al., 2008; Garrigues et al., 2002).

Recently published mechanism is the endocytosis of cyclodextrins (Fenyvesi et al., 2014; Plazzo et al., 2012; Rosenbaum et al., 2010 Wei et al., 2011). These molecules are unable to permeate the cell membrane by diffusion because of their big molecular size and hydrophilic character but cells can take up cyclodextrins by endocytosis. This action was found in different cell types, but the role of this phenomenon in drug absorption is not already known. However, successful DNA delivery was demonstrated with poly-6cationic amphiphilic CD, which formed nanoparticulate complexes. In this case macropinocytosis was responsible for the cellular uptake 3 of transfection complexes (O'Neill et al., 2011). Intracellular activity of cyclodextrins is also demonstrated on Niemann-Pick type C mutant cells by decreasing cholesterol accumulation at the level of endocytotic organelles (Rosenbaum et al., 2010).

Recently we demonstrated that fluorescent random methyl$\beta$-cyclodextrin is able to enter the cytoplasm of Caco- 2 cells by endocytosis (Fenyvesi et al., 2014), but the cellular uptake of different $\beta$-cyclodextrin derivatives has not been investigated yet. The absorption-enhancing effects of cyclodextrins are well known; however, the role of endocytosis in this process has not been studied.

According to the abovementioned results cyclodextrins are able to act inside the cells, thus we aimed to study the permeability and endocytosis of fluorescently labeled (2-hydroxypropyl)- $\beta$-cyclodextrin, random methyl- $\beta$-cyclodextrin and soluble $\beta$-cyclodextrin polymer and their inclusion complexes with fluorescently labeled paclitaxel (Flutax) on Caco-2 cells. We also investigated the possibility of the cellular uptake of Flutax- rhodamine-labeled random methyl- $\beta$-cyclodextrin complex via the endocytotic pathway.

\section{Materials and methods}

\subsection{Materials}

Random methyl- $\beta$-cyclodextrin (RAMEB, DS 12), (2-hydroxypropyl)- $\beta$-cyclodextrin (HPBCD, DS 4.5), soluble $\beta$-cyclodextrin polymer (BCDpolymer, cross-linked with epichlorohydrin, average $\mathrm{MW}=92 \mathrm{kDa}), \quad 6$-deoxy-6-[(5/6)-rhodaminylthioureido]-RAMEB (Rho-RAMEB, DS = 1 for RBITC, DS=12 for methyl), 6-deoxy-6[(5/6)-fluoresceinylthioureido]-RAMEB (FITC-RAMEB, DS $=1$ for
A
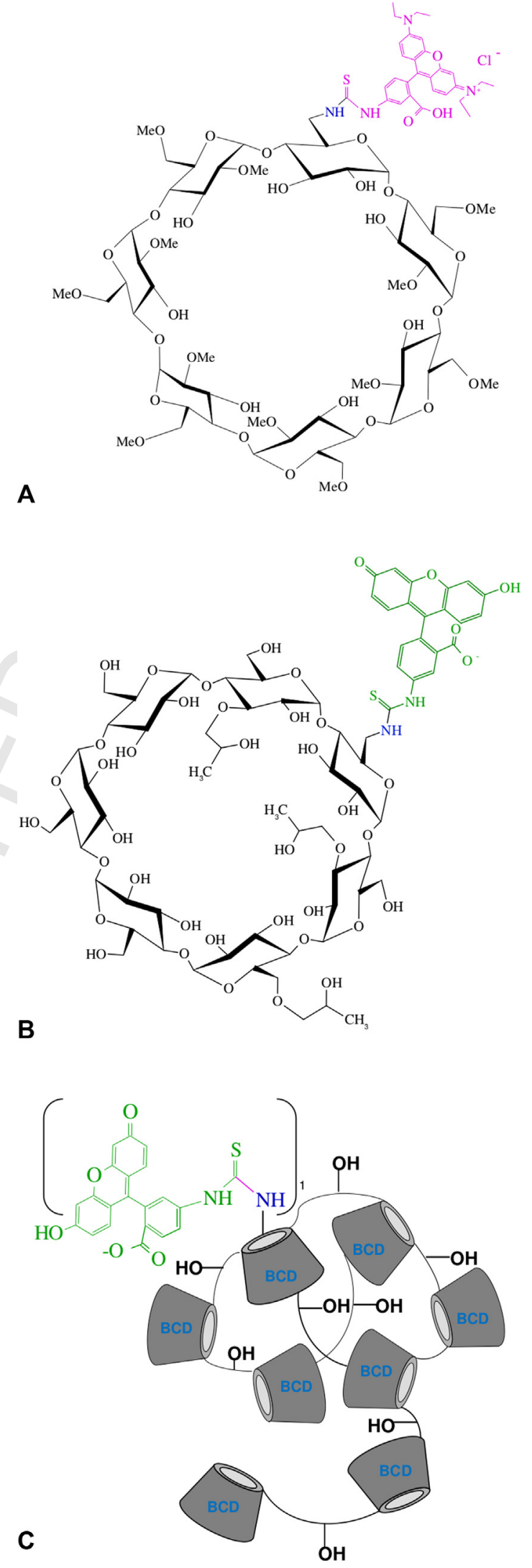

Fig. 1. Chemical structures of fluorescently labeled cyclodextrins. The structure of Rhodamine-labeled RAMEB (A), fluorescein-labeled HPBCD (B) and fluoresceinlabeled $B C D$ polymer $(C)$ are demonstrated in this figure. Fluorescent groups are attached to the cyclodextrin rings by thioureido groups. 
FITC, DS=12 for methyl), 6-deoxy-6-[(5/6)-fluoresceinylthioureido]-HPBCD (FITC-HPBCD, DS $=1$ for FITC, DS $=3$ for HP) and fluorescein-labeled soluble BCDpolymer (FITC-BCDpolymer, crosslinked with epichlorohydrin, average $\mathrm{MW}=39 \mathrm{kDa}$ ) are from Cyclolab Ltd. (Budapest, Hungary). In the case of fluorescent labeled derivatives the fluorophore (5/6 isomeric mixture) is attached on the primary hydroxyl rim of the cyclodextrin, via a chemically stable thioureido group. Each cyclodextrin ring contains a fluorescent unit, except in the case of FITC-BCDpolymer, where the fluorophore-labeled macrocycle content is $\sim 1 \% \mathrm{w} / \mathrm{w}$. The structures of the fluorescent cyclodextrins are shown in Fig. 1. Log $P$ values $(c \log P$ ) were predicted for FITC-HPBCD, Rho-RAMEB and their parent cyclodextrins with MarvinSketch 6.0.2 software.

Fluorescent paclitaxel derivative (Flutax-1) was from Tocris Bioscience (United Kingdom). CellMask Deep Red plasmamembrane stain was from Invitrogen (Budapest, Hungary). Triton X-100 (TX-100) was from Roche Diagnostics GmbH (Mannheim, Germany). All other reagents were purchased from Sigma-Aldrich (Budapest, Hungary).

\subsection{Caco-2 cell culture}

Caco-2 cell line originates from the European Collection of Cell Cultures (ECACC UK). Caco-2 cells were cultured in Dulbecco's modified Eagle's medium (DMEM) supplemented with $10 \%$ heatinactivated fetal bovine serum, $1 \%$ non-essential amino acid and $1 \%$ penicillin-streptomycin solution at $37^{\circ} \mathrm{C}$ in an incubator containing $5 \% \mathrm{CO}_{2}$. The passage number of the cells was between 25 and 40.

For permeability experiments Caco-2 cells were seeded at the density of 200,000 cells/well on Transwell ${ }^{\circledR}$ (Corning Costar, USA) polycarbonate filters (pore size $0.4 \mathrm{~mm}$, surface area $1.12 \mathrm{~cm}^{2}$ ). Culture medium was replaced with fresh medium every two or three days in the inserts. Monolayers were used for the experiments between 20 and 35 days after seeding. The formation of functional epithelial layers was monitored by the development of transepithelial electrical resistance (TEER) and measured with a Millicell-ERS voltohmmeter (Millipore, USA). In permeability experiments TEER values were also measured at the beginning and at the end of sampling to check monolayer integrity and follow the effects of cyclodextrin treatments.

\subsection{Flutax-cyclodextrin complexes}

Flutax-1, HPBCD, RAMEB and BCDpolymer were dissolved in DMSO (dimethyl-sulfoxide) separately, and then the appropriate solutions were mixed together to get $1: 1$ molar ratio for FlutaxHPBCD and Flutax-RAMEB, 1:1/45 molar ratio for Flutax-BCDpolymer. One molecule of the polymer contains $\sim 45$ monomer rings, thus 45 mol Flutax were calculated for 1 mol BCDpolymer. Solution of Flutax-1 without cyclodextrin was also made. Then samples were frozen and dried by lyophilization. The complexes were kept at $-20^{\circ} \mathrm{C}$ until the experiments.

\subsection{Transepithelial permeability test of $C D$ derivates}

Fluorescently labeled cyclodextrin solutions at the concentration of $50 \mu \mathrm{M}$ were used (FITC-RAMEB, FITC-HPBCD, FITC-
BCDpolymer dissolved in Hanks' Balanced Salt solution (HBSS)) in permeability measurements. Caco-2 monolayers were washed twice and pre-incubated with HBSS for $20 \mathrm{~min}$ at $37^{\circ} \mathrm{C}$ and then incubated apically with cyclodextrin solutions for $2 \mathrm{~h}$ at $37^{\circ} \mathrm{C}$. Samples were collected from the basolateral side at 60 and $120 \mathrm{~min}$ and the volume was replenished with HBSS. The monolayers were washed five times with ice cold HBSS and cells were lysed with $1 \%$ Triton X-100 (TX-100). The permeated amount and the cyclodextrin content of the cell lysates were determined by FLUOstar Optima microplate reader (BMG LABTECH, Offenburg, Germany) at $492 \mathrm{~nm}$ excitation and $520 \mathrm{~nm}$ emission wavelength. Cyclodextrin permeation rates across the monolayers were determined from the concentration values. With the formula below the apparent permeability coefficients were calculated:

$P_{\text {app }}=\frac{\mathrm{d} Q}{\mathrm{~d} t} \times \frac{1}{\left(C_{0} \times A\right)}$

$P_{\text {app }}$ : apparent permeability coefficient $(\mathrm{cm} / \mathrm{s}) ; \mathrm{d} Q / \mathrm{d} t$ : permeability rate of substances $(\mathrm{mol} / \mathrm{s}) ; C_{0}$ : initial concentration of the substances in the apical chamber $(\mathrm{mol} / \mathrm{ml}) ; A$ : surface area of the membrane $\left(\mathrm{cm}^{2}\right)$.

\subsection{Flow cytometry}

For these experiments cells were trypsinized, washed twice with HBSS and resuspended at the density of $2 \times 10^{6}$ cells $/ \mathrm{ml}$. Cell suspensions were incubated with the fluorescent cyclodextrin solutions $(50 \mu \mathrm{M})$ for $30 \mathrm{~min}$ at $37^{\circ} \mathrm{C}$ or on ice. At the end of the treatment cells were washed three times with ice cold HBSS and kept on ice until measurements.

We also performed experiments with the macropinocytosis inhibitor rottlerin to verify the endocytotic pathway for the cellular internalization of fluorescent cyclodextrins. We preincubated the cell suspension with rottlerin at the concentration of $10 \mu \mathrm{M}$ for $40 \mathrm{~min}$ at $37^{\circ} \mathrm{C}$, then FITC-HPBCD and Rho-RAMEB $(50 \mu \mathrm{M})$ were added to the cells and incubated for $30 \mathrm{~min}$. Finally, the samples were washed three times with ice cold HBSS and kept on ice until measurements. In both experimental setups propidium-iodide was added to the cells at the concentration of $2 \mu \mathrm{g} / \mathrm{ml}$ to recognize dead cells. Cellular internalization of fluorescent cyclodextrins was analyzed by flow cytometer (FACScan, Becton-Dickinson, Mountain View, CA, USA). Twocolor analysis was performed and viable cells were gated in according to their low intensity propidium iodide (PI) fluorescence. Fluorescent dyes were excited at $488 \mathrm{~nm}$ with an argon laser. The fluorescence emission of FITC was detected via a 530/ $30 \mathrm{~nm}$ band pass filter, while the fluorescence emission of propidium iodide was detected by a $585 / 42 \mathrm{~nm}$ band pass filter. Data were analyzed by BDIS Cellquest (Becton-Dickinson) and WinMDI 2.8 (written by Joseph Trotter; http://facs.scripps.edu/ software.html) software.

\subsection{Fluorescent microscopy}

For microscopic investigations Caco-2 cells were seeded on round glass coverslips at a density of 100,000 cell $/ 2 \mathrm{ml}$ in 12 well plates. After 4 days cells were washed twice with HBSS and then treated with $50 \mu \mathrm{M}$ cyclodextrin solutions (FITC-HPBCD, Rho-

Table 1

$\log P$ predictions of RAMEB, Rho-RAMEB, HPBCD and FITC-HPBCD.

\begin{tabular}{|c|c|c|c|c|}
\hline$c \log P$ & $\begin{array}{l}-1.53 \\
\text { RAMEB (DS = } 14 \text { for methyl) }\end{array}$ & $\begin{array}{l}-2.57 \\
\text { Rho-RAMEB } \\
\text { (DS }=1 \text { for rhodamine, DS = } 12 \text { for methyl) }\end{array}$ & $\begin{array}{l}-11.29 \\
\mathrm{HPBCD}(\mathrm{DS}=3 \text { for } \mathrm{HP})\end{array}$ & $\begin{array}{l}-7.74 \\
\text { FITC-HPBCD } \\
(\mathrm{DS}=1 \text { for FITC, DS = } 3 \text { for HP) }\end{array}$ \\
\hline
\end{tabular}



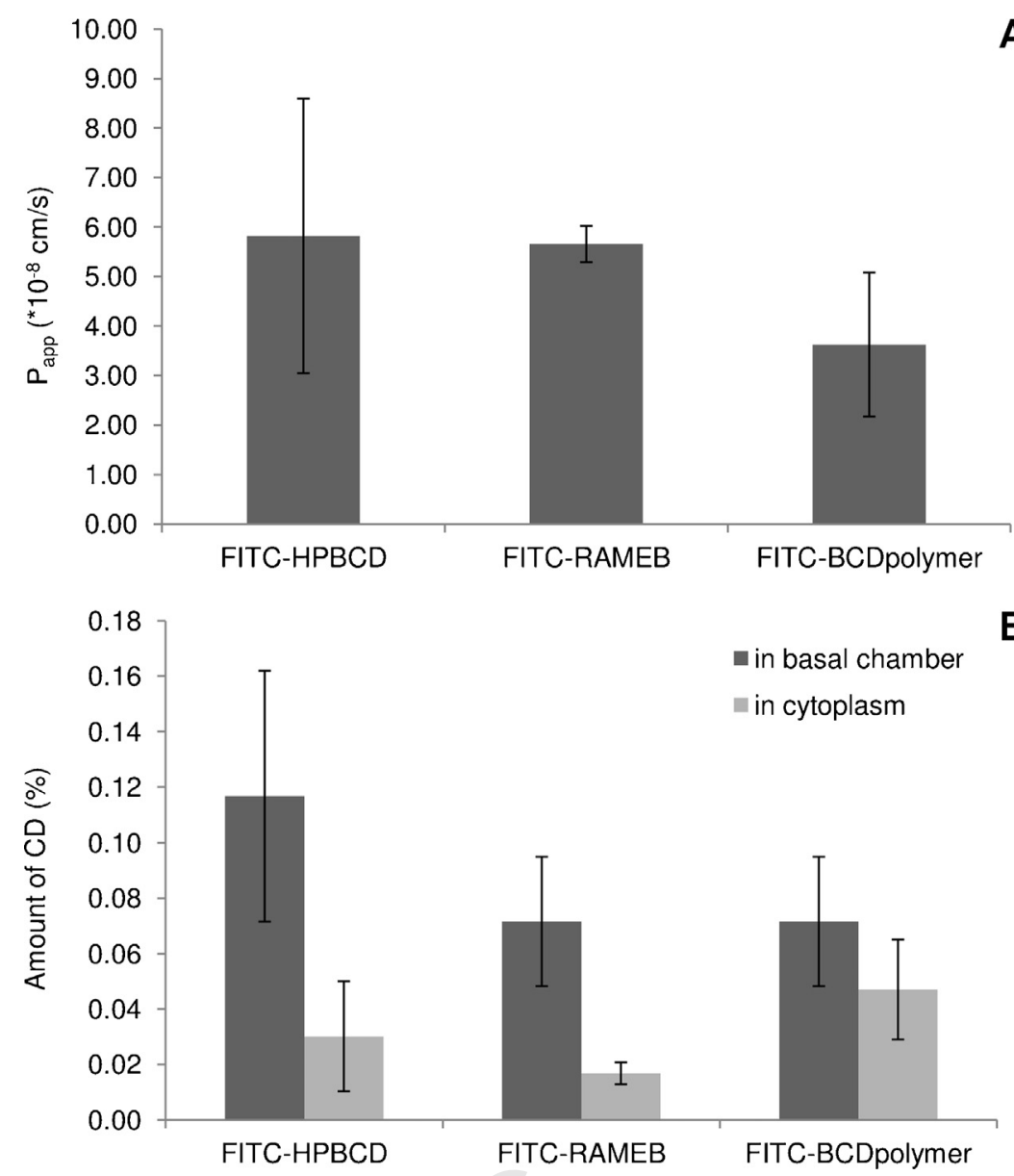

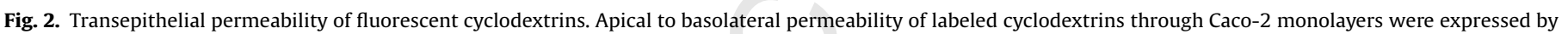

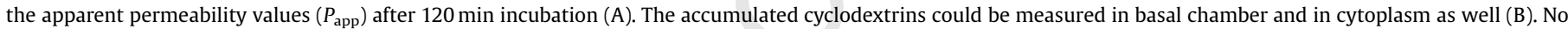

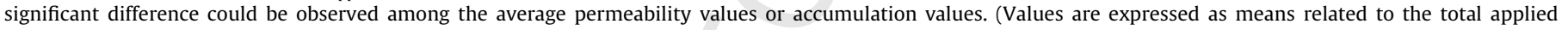
cyclodextrin amount \pm SD, $n=8$ for FITC-HPBCD, $n=6$ for FITC-RAMEB and FITC-BCDpolymer treatments, $p>0.05$ ).

RAMEB and FITC-BCDpolymer). After $30 \mathrm{~min}$, cells were washed 4 times with ice cold HBSS, then fixed with $3 \%$ paraformaldehyde solution. Nuclei were stained with $1 \mu \mathrm{M}$ bis-benzimide solution. At the end coverslips were stuck to microscope slides (Fluoromount). After drying for one hour, samples were investigated under fluorescent microscope (Axio Scope A1, Zeiss).

For confocal microscopy the method was almost the same. There was an extra step before fixation for staining the cell membrane with $1 \mu \mathrm{g} / \mathrm{ml}$ CellMask Deep Red solution. FlutaxRhoRAMEB complex was made by lyophilisation, and it was dissolved in HBSS before the experiment. Samples were investigated under confocal laser scanning microscope.

Optical sections of the cells were recorded with a Zeiss LSM510 confocal microscope using a Plan-Apochromat $63 \times(\mathrm{NA}$ 1.4) oil immersion objective. For the excitation and detection of fluorescent dyes the following laser lines and emission bands were used: FITC, excitation by the $488 \mathrm{~nm}$ line of an Ar ion laser, emission: $505-550 \mathrm{~nm}$; rhodamine excitation by a $543 \mathrm{~nm}$ HeNe laser, emission: 560-615 nm; CellMask Deep Red plasma membrane stain (labeling cell membrane) excitation by a $633 \mathrm{~nm}$ HeNe laser, emission: >650 nm. Line-by-line alternating illumination with the different lasers was used to minimize crosstalk (multi-track mode). $512 \times 512$-pixel images were collected with a pixel size of $0.14 \mu \mathrm{m}$. Image stacks were recorded with a step size of $0.75 \mu \mathrm{m}$ and an optical slice thickness of $0.9 \mu \mathrm{m}$ (pinhole: $125 \mu \mathrm{m}$ ). Images were low-pass filtered to reduce noise.

\subsection{Uptake of Flutax-cyclodextrin complexes}

Caco-2 cells were seeded into a 96-well black plate at a density of 30,000 cells/well. After one week cells formed a monolayer in the plate. Lyophilized cyclodextrin complex samples were dissolved in HBSS at $50 \mu \mathrm{M}$ final concentration. Monolayers were washed twice with HBSS, then treated with the sample solutions for $30 \mathrm{~min}$. After the treatment cells were washed 4 times with ice cold HBSS, and lysed with $1 \%$ TX-100. Flutax content of the cell lysates were determined by FLUOstar Optima microplate reader (BMG LABTECH, Offenburg, Germany) at $492 \mathrm{~nm}$ excitation and $520 \mathrm{~nm}$ emission wavelength.

\subsection{Statistical analysis}

For statistical analysis SigmaStat software (version 3.1; SPSS Inc.) and Excel were used. Data are presented as means \pm S.D. Comparison of two groups was performed by unpaired $t$-test, while comparison of more than two groups was performed using ANOVA. Differences were considered significant at $p<0.05$.

\section{Results}

\subsection{Log P predictions of cyclodextrins}

Predicted octanol-water partition coefficients were very low for fluorescently labeled and unlabeled cyclodextrin derivatives. 

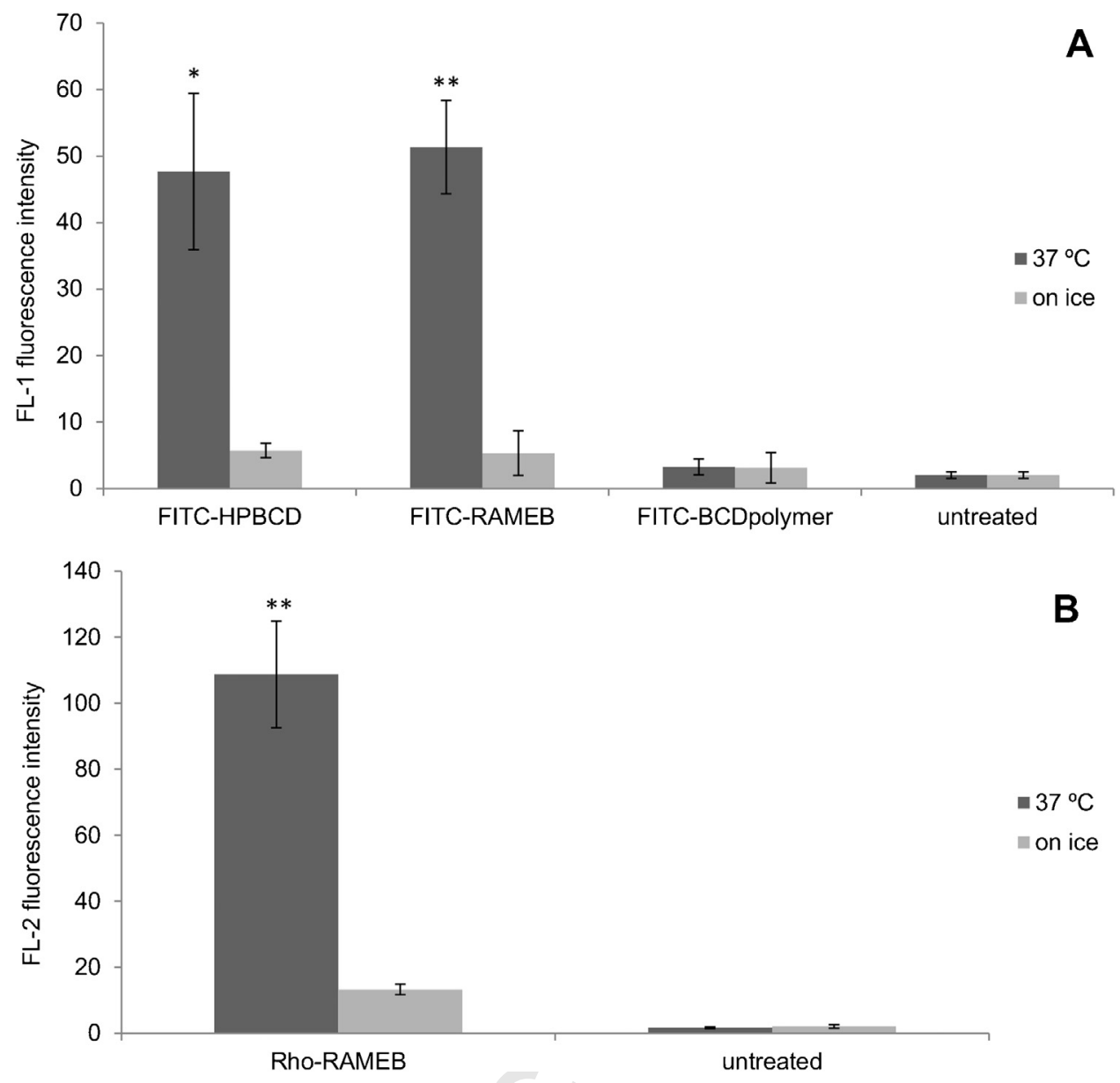

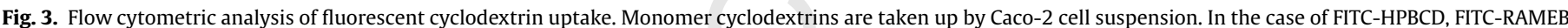

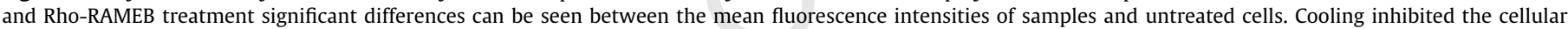

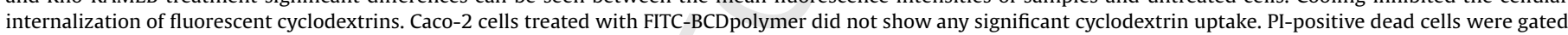
out from data analysis. (Data are expressed as means $\pm \mathrm{SD}, n=3$, significance is expressed as ${ }^{*} p<0.05$ and ${ }^{* *} p<0.01$ ).

HPBCD and FITC-HPBCD have much lower $c \log P$ value than RAMEB and Rho-RAMEB (Table 1).

\subsection{Transepithelial permeability test of $C D$ derivates}

In order to investigate the permeability of the fluorescent derivatives of RAMEB, HPBCD and BCDpolymer through the intestinal epithelial barrier we applied Caco-2 monolayers. Caco2 absorption model is excellent to investigate passive and active mechanisms, which are involved in drug absorption. Cyclodextrin solutions were used at a concentration of $50 \mu \mathrm{M}$. The permeability of cyclodextrin derivatives was determined and the results were expressed in apparent permeability values $\left(P_{\text {app }}\right)$. The apparent permeabilities of fluorescent cyclodextrins were very low, and there

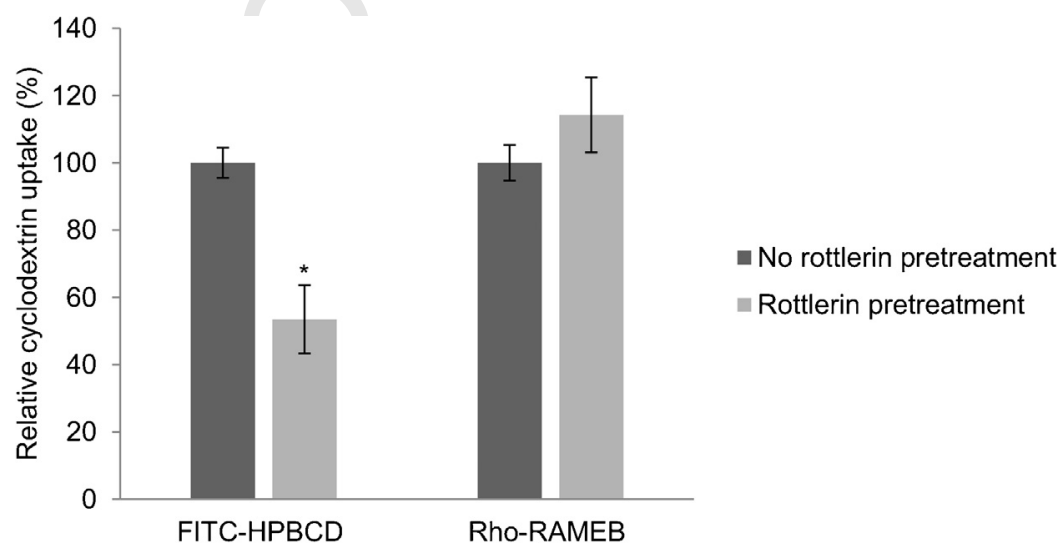

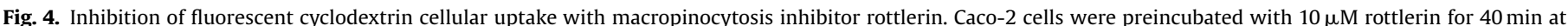

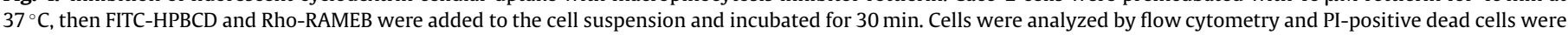

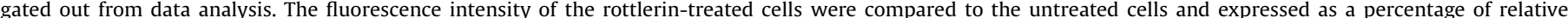

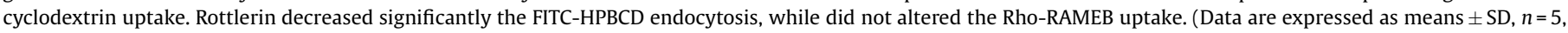
significance is expressed as ${ }^{*} p<0.05$ ). 


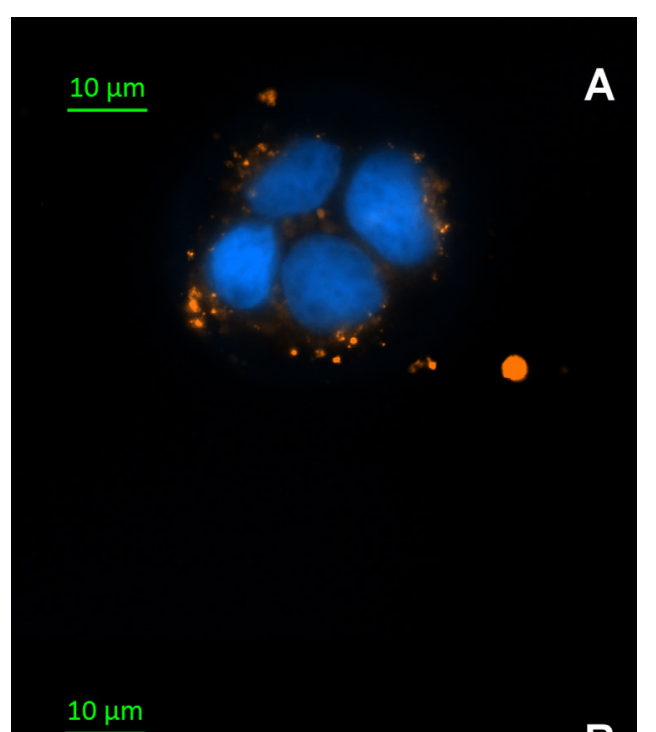

B

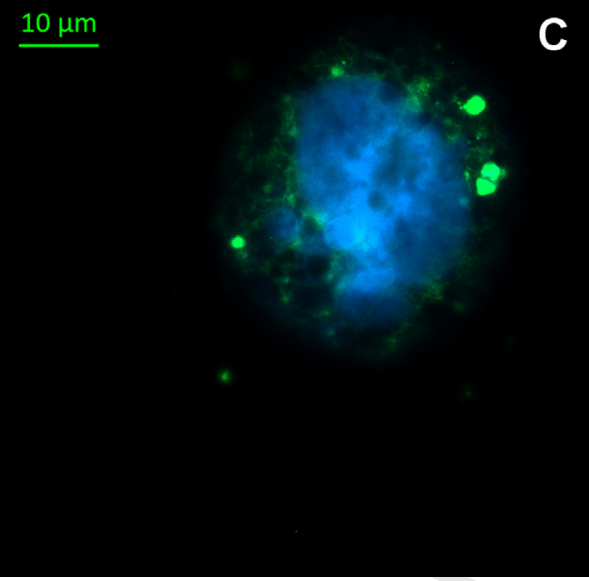

Q6 Fig. 5. Fluorescent microscopic images of labeled cyclodextrins in Caco-2 cells. The distribution of different cyclodextrin derivatives in the cytoplasm of Caco-2 cells can be seen in endocytotic vesicles. Rho-RAMEB is localized in large (red) vesicles (A), small (green) endosomes can be detected in the case of FITC-HPBCD (B), and FITC-BCDpolymer was taken up into the largest (green) endosomes (C). Cell nuclei are labeled with blue bis-benzimide staining. (For interpretation of the references to color in this figure legend, the reader is referred to the web version of this article.)

were no significant differences among the values. They were 5.82 $\pm 2.8 \times 10^{-8} \mathrm{~cm} / \mathrm{s}, \quad 5.65 \pm 0.36 \times 10^{-8} \mathrm{~cm} / \mathrm{s}$ and $3.62 \pm 1.45 \times 10^{-8}$ $\mathrm{cm} / \mathrm{s}$ for FITC-HPBCD, FITC-RAMEB and FITC-BCD Polymer, respectively $(p>0.05)$ (Fig 2A). The integrity of monolayers did not change after cyclodextrin treatments, according to TEER values. In Fig. 2B the accumulated amount of labeled cyclodextrins can be seen in the basal chamber and in the cytoplasm. Because of the low permeability there were limited amounts of CDs in the basal chambers, and also in the cytoplasm. These amounts were measurable by a microplate reader, but the difference of the results of the CDs was not significant in either cases.
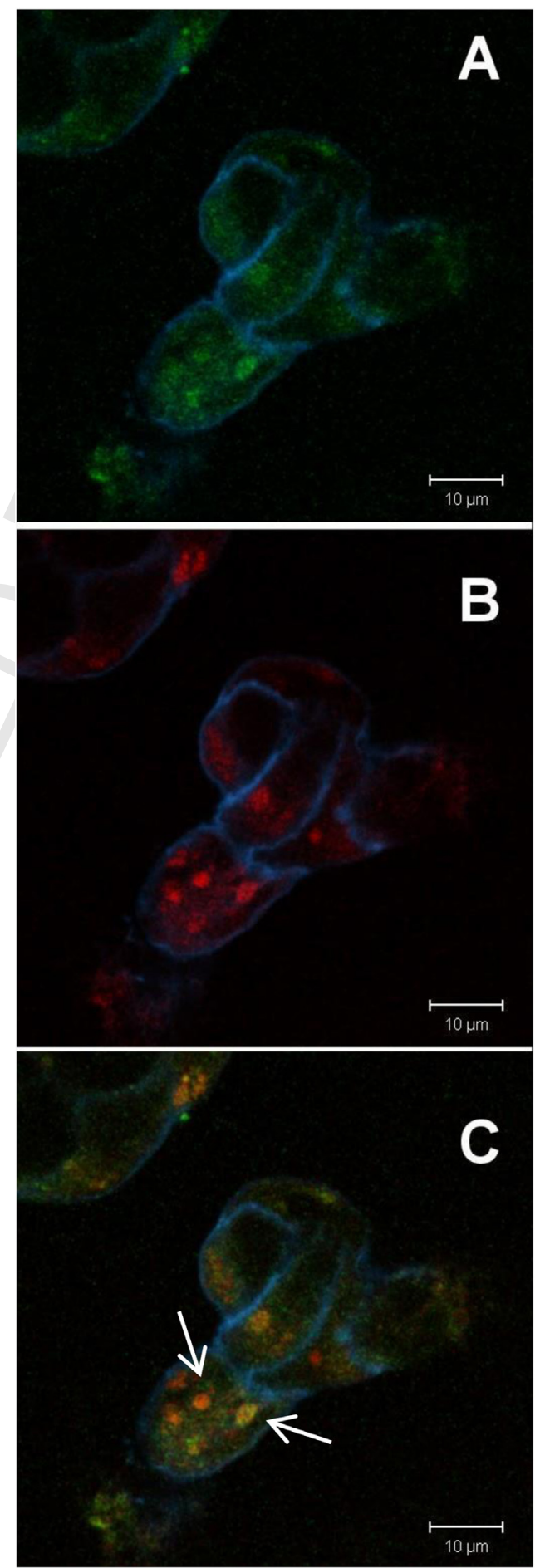

Fig. 6. Confocal microscopic images of Flutax-Rho-RAMEB complexes in Caco2 cells. Caco- 2 cells were treated with Flutax-Rho-RAMEB complex for $30 \mathrm{~min}$ and cytoplasm shows almost the same pattern as in the case of Rho-RAMEB treatment. Vesicles containing Flutax (green) and Rho-RAMEB (red) can be detected separately, but there are vesicles, which contains both molecules (orange). Cell membrane is indicated with blue. Pictures were taken on cell membrane and Flutax (A), cell membrane and Rho-RAMEB (B) and all three channels together (C). (For interpretation of the references to color in this figure legend, the reader is referred to the web version of this article.)

Please cite this article in press as: Réti-Nagy, K., et al., Endocytosis of fluorescent cyclodextrins by intestinal Caco-2 cells and its role in paclitaxel drug delivery. Int J Pharmaceut (2015), http://dx.doi.org/10.1016/j.ijpharm.2015.10.049 


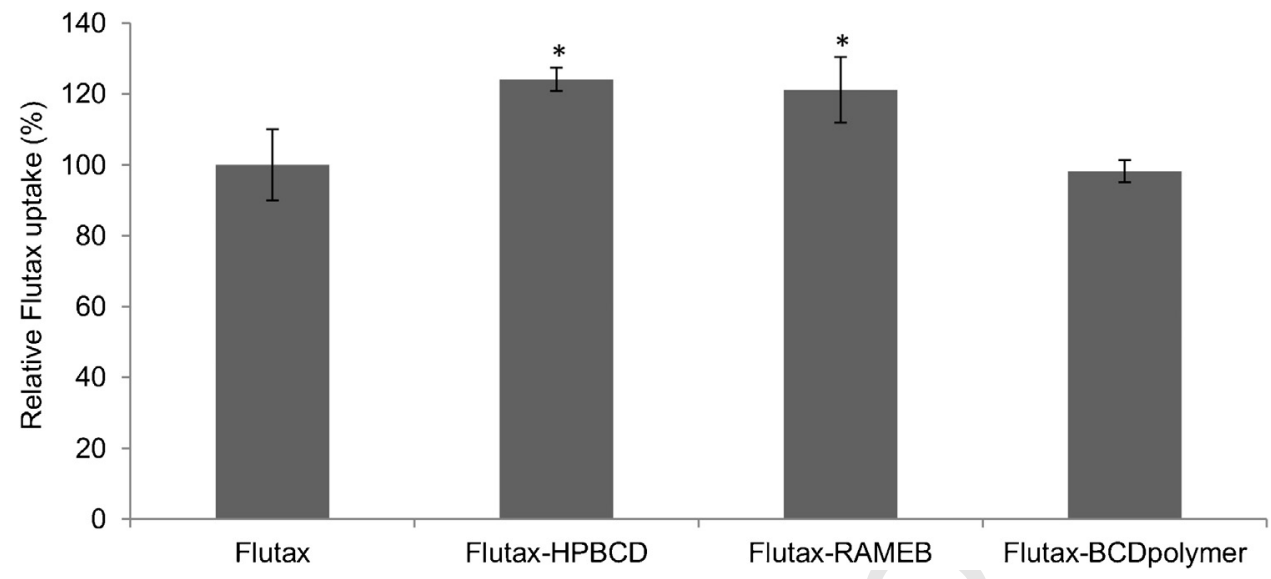

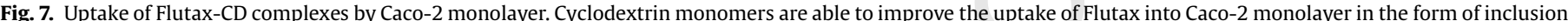

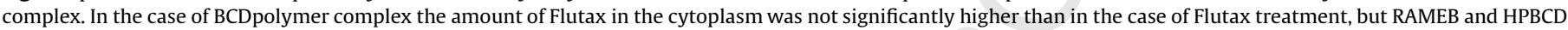
complexes significantly improved Flutax uptake. (Data are expressed as means $\pm \operatorname{SD}, n=9$, significance is expressed as $* p<0.05$ ).

\subsection{Flow cytometry}

After gating viable cells according to their low propidium iodine fluorescence intensity, the amounts of intracellular cyclodextrin were evaluated. Only FITC-HPBCD, FITC-RAMEB and Rho-RAMEB treatment $\left(37^{\circ} \mathrm{C}, 30 \mathrm{~min}\right)$ caused significant fluorescence intensity increment in Caco- 2 cell suspension, compared also to untreated cells $(p<0.05)$ (Fig. 3). Interestingly FITC-BCDpolymer had significantly lower intracellular fluorescence at the same concentration. It should be noted that the labeling process is different and the fluorophore ratio is much smaller in the case of the polymer.

Rottlerin pretreatment at the concentration of $10 \mu \mathrm{M}$, significantly decreased FITC-HPBCD internalization $(p<0.05)$, but interestingly did not decreased Rho-RAMEB cellular uptake (Fig. 4). FITC-BCDpolymer was not investigated in macropinocytosis inhibition experiments by flow cytometry, because the endocytosis of this derivative could not be detected at the applied concentration in cell suspensions.

\subsection{Fluorescent microscopy of fluorescently labeled cyclodextrin derivates}

In this experiment Rho-RAMEB, FITC-HPBCD and FITC-BCDpolymer were used to demonstrate that these cyclodextrins are able to enter Caco-2 cells. Each cyclodextrin derivative can be detected in the cytoplasm in smaller or larger endocytotic vesicles (Fig. 5). FITC-HPBCD has the most homogenous distribution (Fig 5B); RhoRAMEB can be found in larger endosomes (Fig 5A) and in the case of FITC-BCDpolymer the largest vesicles can be detected (Fig 5C). These differences are probably due to the different substituents and molecular properties of the derivates.

\subsection{Confocal microscopy of Flutax-Rho-RAMEB complex}

Confocal microscopy was used to detect colocalization of Flutax and Rho-RAMEB in endocytotic vesicles. A single optical section displaying vesicles containing both molecules (Flutax: green, cyclodextrin: red) is shown in Fig. 6. Both compounds are present in the cytoplasm in a diffuse form as well.

\subsection{Uptake of Flutax-cyclodextrin Complexes by Caco-2 Monolayer}

To determine the absorption increasing effect of the investigated cyclodextrins, lyophilized complexes were used with unlabeled cyclodextrins. After 30 -minute incubation $\left(37^{\circ} \mathrm{C}\right)$ the cell lysates showed higher fluorescence intensity in the case of Flutax-HPBCD and Flutax-RAMEB. These two types of complexes increased the uptake of Flutax-1 into Caco- 2 monolayer by around $20 \%$. This effect was significant in both cases $(p<0.05)$. Complexation with BCDpolymer did not improve Flutax-1 absorption (Fig. 7).

\section{Discussion}

Some cyclodextrins have been included in various pharmacopoeias as solubilizers and absorption enhancers for long, but recently HPBCD itself has also been recognized as orphan drug for the treatment of Niemann-Pick type C1 disease (Matsuo et al., 2013; Ottinger et al., 2014). RAMEB is a conventionally applied methylated $\beta-C D$ with excellent complexing abilities and strong cellular toxicity (Kiss et al., 2010), while the cellular effects of BCDpolymer have not been studied widely. In general, it is difficult to study directly the mechanism of cyclodextrin-cell membrane interactions and the fate of cyclodextrins in biological systems. The development of fluorescent cyclodextrin derivatives provides valuable tools and opens new perspectives to reveal the cellular effects of cyclodextrins.

An unprecedented phenomenon, the endocytosis of cyclodextrins has been revealed recently by the help of fluorescent derivatives on several cell types. We also found in our previous study that intestinal Caco-2 cells could take up FITC-RAMEB into their cytoplasm by macropinocytosis (Fenyvesi et al., 2014). In the present study we involved other cyclodextrin derivatives and the behavior of fluorescently labeled HPBCD and BCDpolymer was investigated on Caco-2 cells compared to fluorescently labeled RAMEB. Caco-2 model is suitable to study the effects of excipients on drug absorption, and in our experiments the interaction of cyclodextrins with the intestinal epithelial barrier could be observed. Furthermore, we aimed to investigate the uptake of these cyclodextrin derivatives complexed with fluorescently labeled paclitaxel on Caco- 2 cells.

At first the permeability test of the cyclodextrins was performed on Caco-2 monolayers, and in accordance with our previous results, very low and not significantly different apparent permeability values were measured for all the studied derivatives (FITC-HPBCD, FITC-RAMEB and FITC-BCDpolymer). Surprisingly, all three types of labeled cyclodextrins could be detected both in the basal chamber and in the cytoplasm. It was confirmed by fluorescent microscopy that after $30 \mathrm{~min}$ of incubation all the 
labeled derivatives could be detected in attached cells. FITC$\mathrm{HPBCD}$, FITC-BCDpolymer and Rho-RAMEB were found in the cytoplasm in vesicles of different size.

Nevertheless, flow cytometry results showed that in the case of Caco- 2 cell suspension only the monomer derivatives (FITC-HPBCD and FITC-RAMEB) were detectable in the cells after $30 \mathrm{~min}$ of incubation at $37^{\circ} \mathrm{C}$. This uptake could be inhibited by keeping samples on ice and with rottlerin pretreatment. However RhoRAMEB internalization could not be inhibited by rottlerin. In our previous publication (Fenyvesi et al., 2011) we demonstrated that rottlerin decreased the endocytosis of FITC-RAMEB. These results raise the possibility that FITC and Rhodamine derivatives of methylated $\beta$-cyclodextrins are internalized by different processes.

Cyclodextrins are hydrophilic molecules with low octanolwater partition coefficients $(\log P$ ) (Kurkov and Loftsson, 2012; Loftsson, 2015). The fluorescein and rhodamine labeling increased their molecular weight and altered the properties of the parental cyclodextrins, but they kept their good water solubility. We predicted $\log P$ values $(c \log P$ ) for FITC-HPBCD, Rho-RAMEB and their parent cyclodextrins and found that fluorescent derivatives retained their hydrophilicity. These data confirm that fluorescent cyclodextrins are not able to cross cell membrane by passive diffusion, similarly to the unlabeled cyclodextrins.

Finally, paclitaxel-cyclodextrin complexes were investigated on Caco-2 cell layers. It was clearly shown that the complexes of RAMEB and HPBCD were able to increase Flutax uptake in Caco2 monolayers, while BCDpolymer had no effect on it. It is in accordance with our previous finding, where we have shown that RAMEB and its derivatives are able to enhance paclitaxel permeability on Caco-2 monolayers (Fenyvesi et al., 2011). In our present study we also investigated the uptake of fluorescent complexes at cellular level and intracellular colocalization of the fluorescent paclitaxel derivative, Flutax-1 and Rho-RAMEB could be identified. We demonstrated for the first time that fluorescent cyclodextrins entered the cells through endocytotic pathways with a highly lipophilic substrate and host-guest molecules could be detected together in an intracellular endosome.

Based on the above mentioned results and the widely known properties of cyclodextrins $\beta$-cyclodextrins have multiple effects on intestinal barrier:

(i) the enhancement of water solubility of lipophilic drugs,

(j) enhanced permeation of lipophilic molecules through the unstirred water layer (UWL),

(k) permeabilization of cell membrane by removing cholesterol, which leads to further consequences such as,

(1) changes in the function of tight junctions, with increased paracellular permeability and,

(m) inhibition of efflux pumps,

(n) endocytosis of free cyclodextrins,

(o) endocytosis of cyclodextrin-drug complexes.

The first five mechanisms have been widely studied. In the majority of cases solubility enhancement and the improvement of drug permeability through UWL and cell membrane are the main absorption-enhancing factors. However, some membrane permeation-limiting factors such as the molecular size, the complex stability and the presence of efflux pumps may counterbalance and in certain cases even cyclodextrins can enter the cells by endocytosis. Endocytosis of CD complexes has usually less significance in the case of membrane penetrable small molecules, but strong binding forces can overwrite this hypothesis: the nondissociating host-guest assembly can enter the cells. Paclitaxel, our model drug is a poorly soluble, but membrane penetrable molecule. It is also a P-gp substrate which limits its cellular uptake. It is well known that paclitaxel forms complexes with $\beta$-cyclodextrins both in aqueous solutions and in nanoparticles (Agueros et al., 2009; Bouquet et al., 2007; Szente et al., 1999). Paclitaxel-RAMEB complexes have medium-to-high stability constants $\left(K_{1: 1}=4850 \mathrm{M}^{-1}\right)$ (Szente et al., 2001); thus, it has a chance to enter the cell by fluid phase endocytosis as a stable unit. The enhanced size of the molecular assembly of the inclusion complex can also provoke the endocytosis of the complex.

Usually the big molecules, such as peptides or oligonucleotides, cannot penetrate the cell membrane. In this case a possible route is the endocytotic pathway to overcome the barrier of cell membrane. Similarly, endocytosis of the large and hydrophilic CD-drug complexes is also conceivable. The failure of the CDpolymer as penetration enhancer of paclitaxel might be the lower complexing affinity due to the high degree of polymerization (low accessibility of the $\mathrm{CD}$ rings for paclitaxel).

Although the exact mechanism has not been revealed as yet, the cyclodextrin rings are optimal drug carriers because they can be modified by various substituents in order to obtain the best carrier for stimulating the internalization through different endocytotic pathways.

\section{Conclusions}

Various fluorescent cyclodextrin derivatives are able to enter Caco-2 intestinal cells by endocytosis. We demonstrated for the first time that $\beta$-cyclodextrins can improve the bioavailability of drugs with poor solubility and absorption not only by solubility improvement but by transporting the complexed drug into the cytoplasm of enterocytes via endocytosis. The permeability and absorption enhancing effect of cyclodextrins might involve several mechanisms, which may act simultaneously and thus, it is difficult to examine separately in cellular systems. However, we emphasize that in some special cases also endocytotic processes should be considered.

\section{Acknowledgements}

This research was supported by the European Union and the State of Hungary, co-financed by the European Social Fund in the framework of TÁMOP 4.2.4. A/2-11-1-2012-0001 'National Excellence Program'. The research was also supported by the following grants: Marie Curie Initial Training Programme (ITN-237962/ 2008), Hungarian Scientific Research Fund (OTKA K103965), TÁMOP422-2012-0023VÉD-ELEM implemented through the New Hungary Development Plan co-financed by the European Social Fund and the European Regional Development Fund.

\section{References}

Agueros, M., Ruiz-Gaton, L., Vauthier, C., Bouchemal, K., Espuelas, S., Ponchel, G., Irache, J.M., 2009. Combined hydroxypropyl-beta-cyclodextrin and poly (anhydride) nanoparticles improve the oral permeability of paclitaxel. Eur. J. Pharm. Sci. 38, 405-413.

Arima, H., Yunomae, K., Morikawa, T., Hirayama, F., Uekama, K., 2004. Contribution of cholesterol and phospholipids to inhibitory effect of dimethyl-betacyclodextrin on efflux function of $P$-glycoprotein and multidrug resistanceassociated protein 2 in vinblastine-resistant Caco- 2 cell monolayers. Pharm. Res. 21, 625-634.

Bouquet, W., Ceelen, W., Fritzinger, B., Pattyn, P., Peeters, M., Remon, J.P., Vervaet, C., 2007. Paclitaxel/beta-cyclodextrin complexes for hyperthermic peritoneal perfusion - formulation and stability. Eur. J. Pharm. Biopharm. 66, 391-397.

Brewster, M.E., Noppe, M., Peeters, J., Loftsson, T., 2007. Effect of the unstirred water layer on permeability enhancement by hydrophilic cyclodextrins. Int. J. Pharm. $342,250-253$.

Fenyvesi, F., Fenyvesi, E., Szente, L., Goda, K., Bacso, Z., Bacskay, I., Varadi, J., Kiss, T., Molnar, E., Janaky, T., Szabo, G.J., r, Vecsernyes, M., 2008. P-glycoprotein inhibition by membrane cholesterol modulation. Eur. J. Pharm. Sci. 34, 236-242.

Fenyvesi, F., Kiss, T., Fenyvesi, E., Szente, L., Veszelka, S., Deli, M.A., Varadi, J., Feher, P., Ujhelyi, Z., Tosaki, A., Vecsernyes, M., Bacskay, I., 2011. Randomly methylated 
G Model

IJP 15304 1-9

K. Réti-Nagy et al./International Journal of Pharmaceutics xxx (2015) xxx-xxx

9

484

486

487

488

490

491

492

493

494

495

496

497

498

499

500

501

502

503

505

506

507

508

509 beta-cyclodextrin derivatives enhance taxol permeability through human intestinal epithelial Caco-2 cell monolayer. J. Pharm. Sci. 100, 4734-4744.

Fenyvesi, F., Reti-Nagy, K., Bacso, Z., Gutay-Toth, Z., Malanga, M., Fenyvesi, E., Szente, L., Varadi, J., Ujhelyi, Z., Feher, P., Szabo, G., Vecsernyes, M., Bacskay, I., 2014. Fluorescently labeled methyl-beta-cyclodextrin enters intestinal epithelial Caco-2 cells by fluid-phase endocytosis. PLoS One 9, e84856.

Garrigues, A., Escargueil, A.E., Orlowski, S., 2002. The multidrug transporter, $P$ glycoprotein, actively mediates cholesterol redistribution in the cell membrane. Proc. Natl. Acad. Sci. U. S. A. 99, 10347-10352.

Kiss, T., Fenyvesi, F., Bacskay, I., Varadi, J., Fenyvesi, E., Ivanyi, R., Szente, L., Tosaki, A., Vecsernyes, M., 2010. Evaluation of the cytotoxicity of beta-cyclodextrin derivatives: evidence for the role of cholesterol extraction. Eur. J. Pharm. Sci. 40 , 376-380.

Kurkov, S.V., Loftsson, T., 2012. Cyclodextrins. Int. J. Please provide complete bibliography details for Refs. "Kurkov and Loftsson, 2012and Szente etal., 2001". Pharm..

Lennernas, H., 1998. Human intestinal permeability. J. Pharm. Sci. 87, 403-410.

Loftsson, T., 2015. Excipient pharmacokinetics and profiling. Int. J. Pharm. 480, 4854.

Loftsson, T., Jarho, P., Masson, M., Jarvinen, T., 2005. Cyclodextrins in drug delivery. Expert Opin. Drug Deliv. 2, 335-351.

Loftsson, T., Vogensen, S.B., Brewster, M.E., Konradsdottir, F., 2007. Effects of cyclodextrins on drug delivery through biological membranes. J. Pharm. Sci. 96 , 2532-2546.

Masson, M., Loftsson, T., Masson, G., Stefansson, E., 1999. Cyclodextrins as permeation enhancers: some theoretical evaluations and in vitro testing. J. Controlled Release 59, 107-118.

Matsuo, M., Togawa, M., Hirabaru, K., Mochinaga, S., Narita, A., Adachi, M., Egashira, M., Irie, T., Ohno, K., 2013. Effects of cyclodextrin in two patients with NiemannPick Type C disease. Mol. Genet. Metab. 108, 76-81.
O’Neill, M.J., Guo, J., Byrne, C., Darcy, R., O’Driscoll, C.M., 2011. Mechanistic studies on the uptake and intracellular trafficking of novel cyclodextrin transfection complexes by intestinal epithelial cells. Int. J. Pharm. 413, 174-183.

Ottinger, E.A., Kao, M.L., Carrillo-Carrasco, N., Yanjanin, N., Shankar, R.K., Janssen, M., Brewster, M., Scott, I., Xu, X., Cradock, J., Terse, P., Dehdashti, S.J., Marugan, J., Zheng, W., Portilla, L., Hubbs, A., Pavan, W.J., Heiss, J., CHV, Walkley, S.U., Ory, D. S., Silber, S.A., Porter, F.D., Austin, C.P., McKew, J.C., 2014. Collaborative development of 2-hydroxypropyl-beta-cyclodextrin for the treatment of Niemann-Pick type C1 disease. Curr. Top. Med. Chem. 14, 330-339.

Plazzo, A.P., Hofer, C.T., Jicsinszky, L., Fenyvesi, E., Szente, L., Schiller, J., Herrmann, A., Muller, P., 2012. Uptake of a fluorescent methyl-beta-cyclodextrin via clathrindependent endocytosis. Chem. Phys. Lipids 165, 505-511.

Ren, B., Gao, H., Cao, Y., Jia, L., 2015. In silico understanding of the cyclodextrinphenanthrene hybrid assemblies in both aqueous medium and bacterial membranes. J. Hazard. Mater. 285, 148-156.

Rosenbaum, A.I., Zhang, G., Warren, J.D., Maxfield, F.R., 2010. Endocytosis of betacyclodextrins is responsible for cholesterol reduction in Niemann-Pick type C mutant cells. Proc. Natl. Acad. Sci. U. S. A. 107, 5477-5482.

Stella, V.J., He, Q., 2008. Cyclodextrins. Toxicol. Pathol. 36, 30-42.

Szejtli, J., 2004. Past, present, and future of cyclodextrin research. Pure Appl. Chem. 76, 1825-1845.

Szente, L., Szejtli, J., Vikmon, A, 2001. Inclusion complexes of taxol or taxotere or taxus extract formed with cyclodextrins, its preparation and use.

Szente, L., Vikmon, M., Szeman, J., Otta, K., 1999. Methyl-beta-cyclodextrin/ paclitaxel aqueous solutions: a tool to test Cremophor-free paclitaxel. Stp Pharm. Sci. 9, 243-247.

Wei, H., Zheng, W., Diakur, J., Wiebe, L.I., 2011. Confocal laser scanning microscopy (CLSM) based evidence for cell permeation by mono-4-(N-6-deoxy-6-aminobeta-cyclodextrin)-7-nitrobenzofuran (NBD-beta-CyD). Int. J. Pharm. 403, 1522.

510
511
512
513
514
515

516
517
518
519
520
521
522
523
524
525
526
527
528
529
530
531
532
533
534
535
536
537
538

Please cite this article in press as: Réti-Nagy, K., et al., Endocytosis of fluorescent cyclodextrins by intestinal Caco-2 cells and its role in paclitaxel

drug delivery. Int J Pharmaceut (2015), http://dx.doi.org/10.1016/j.ijpharm.2015.10.049 